\title{
Afferent Perturbations during "Monopodal" Swimming Movements in the Turtle: Phase-dependent Cutaneous Modulation and Proprioceptive Resetting of the Locomotor Rhythm ${ }^{1}$
}

\author{
PAUL R. LENNARD \\ Department of Biology, Emory University, Atlanta, Georgia 30322
}

\begin{abstract}
Locomotion consists of a repeating series of movement cycles (locomotor rhythm) with an orderly activation of musculature during each movement cycle (intracycle motor pattern). The effects of sensory stimulation, on both the intracycle motor pattern and the locomotor rhythm, were examined during electrically elicited swimming movements of a single turtle hindlimb. The resulting "monopodal" swimming was not subject to movement-related reflexes from other limbs or postural constraints, and provided a sensitive system for analyzing the effects of transient sensory perturbations.

During "monopodal" swimming, cutaneous and extensor muscle-nerve stimulation (single 0.1 - to $0.3-\mathrm{msec}$ electrical pulse) had similar phase-dependent effects on the swim cycle in progress. Stimuli delivered during the powerstroke (limb retracting) shortened the period of the cycle. Stimulation during the returnstroke prolonged the cycle. Changes in cycle period were accompanied by in-phase adjustments of the EMG burst duration or interburst interval which was being expressed at the time of stimulus delivery. The in-phase adjustment of each muscle served to maintain the timing relationships between muscles, and resulted in the preservation of the intracycle motor pattern.

Cutaneous and muscle-nerve stimulation had dramatically different effects on the locomotor rhythm. Cutaneous nerve stimulation produced period changes in poststimulus cycles which led to a temporary phase shift of the swimming rhythm. This temporary modulation suggests that cutaneous afferents do not have direct access to the timing circuitry of the central nervous system locomotor network. Muscle-nerve stimulation only altered the period of the cycle in progress at the time of stimulus delivery, and thus permanently reset the locomotor rhythm. This permanent phase shift suggests that muscle afferents have direct access to a central timing network which controls the locomotor rhythm.
\end{abstract}

Received June 18, 1984; Revised December 28, 1984; Accepted December 281984

${ }^{1}$ This work was supported by National Institutes of Health Grant NS 17732. I wish to thank A. W. English for many helpful discussions and assistance with computer programs, D. L. Phillips for developing nonparametric statistical tests, J. Hermanson for drawing Figure 1, L. Reid for technical assistance, and $J$. Hermanson, P. S. G. Stein, and D. R. Stokes for their critical reading of an early version of the manuscript.
The neural control of locomotion requires an effective interaction between the intrinsic locomotor capabilities of the spinal cord and the segmental, crossed spinal, propriospinal, and supraspinal inputs which modify the locomotor output (Grillner, 1975; Stein, 1978; Wetzel and Stuart, 1976). Various roles for these inputs to the spinal locomotor network have been suggested which include: initiation, maintenance, and frequency control of the locomotor rhythm (Shik et al., 1966); reinforcement of the locomotor rhythm (e.g., Andersson and Grillner, 1983); interlimb coordination (e.g., English and Lennard, 1982); retraction/protraction transitions within a locomotor cycle (Pearson and Duysens, 1976; Grillner and Rossignol, 1978); and stumbling corrective reactions (Forssberg, 1979).

Recently, considerable attention has been focused on transient segmental and crossed spinal inputs which are capable of exerting a phase-dependent effect during locomotion. The term, phasedependent effect, refers to the observation that the nature of the efferent adjustment will vary as a function of the timing of the afferent stimulus within the locomotor cycle. These efferent adjustments can result in changes in the amplitude and/or duration of activity in specific muscles during the locomotor cycle in progress at the time of the input (perturbed cycle). Little information, however, is available on the influence of transient phase-dependent effects on the timing relationships between muscles (intracycle motor pattern) within the perturbed and subsequent cycles. At present, there is also only limited information, provided by Duysens (1977), on long-term changes in the locomotor rhythm following transient phase-dependent sensory inputs.

Most studies of the phase-dependent modulation of cat stepping have employed tactile or electrical stimulation of cutaneous afferents. While phase-dependent cutaneous effects were initially described in the chronic spinal cat (Forssberg et al., 1975, 1977), similar results have been obtained when supraspinal pathways are intact (e.g., Duysens and Loeb, 1980; Matsukawa et al., 1982; Wand et al., 1980). In these studies, phase-dependent segmental modulation may have been influenced by postural constraints and movementrelated sensory input from the contralateral limb (see Rossignol et al., 1981). Phase-dependent cutaneous modulation, in the absence of all movement-related sensory input, has been demonstrated in the spinal, curarized cat during "fictive locomotion" (Andersson et al., 1978). Previous studies have not, however, utilized a preparation in which it was possible to examine the phase-dependent effects of segmental cutaneous inputs under conditions where the movement of a single, normally afferented, limb are expressed in the absence of movement-related afferent information from other limbs.

Several studies have examined the phase-dependent modulation of cat stepping in response to transient proprioceptive inputs. Andersson and Grillner (1981) have shown alterations in the efferent output during "fictive locomotion" which depended on the direction and delivery time of imposed hip movements. There is also evidence for modulation of la postsynaptic potentials (Schomberg and Beh- 
rends, 1978) and the stretch reflex (Akazawa et al., 1982) during locomotion. However, Duysens and Stein (1978) found that, while phase-dependent cutaneous modulation was present in the intact cat, stimulation of a purely muscle-nerve in the same preparation had little effect during the locomotor cycle. If muscle-nerve stimulation does produce phase-dependent efferent adjustments, the effect may be masked in the intact cat preparation by crossed limb and/ or postural influences.

This report, which utilizes swimming in the intact turtle as an experimental preparation, compares the effects of electrical stimulation of muscle and cutaneous afferents on the perturbed locomotor cycle and the locomotor rhythm. Turtle swimming provides a particularly favorable system since the animal can be fixed in space without hindering the normal swimming movements of the limb in a buoyant medium. It is also possible to elicit locomotor movement of a single hindlimb electrically. The resulting "monopodal" locomotion, with minimal postural constraints, no movement-related reflexes from the other limbs, and no curare-related changes in muscle tone or spindle bias, provides a system which is particularly suited to uncovering the effects of transient afferent perturbations which might be obscured in other preparations. Evidence will be presented which indicates that transient muscle-nerve stimulation can produce a phase-dependent effect which alters the swimming rhythm. The finding of substantially different effects of cutaneous versus muscle afferent input on the locomotor rhythm will be discussed in terms of differential access of these inputs to a proposed spinal locomotor circuit which is functionally separated into central timing and intracycle pattern-generating networks. This work has previously appeared in abstract form (Lennard, 1983).

\section{Materials and Methods}

\section{Surgery and electrode implantation}

Red-eared turtles (Pseudemys scripta elegans; $n=53$ ), with a carapace length of 15 to $25 \mathrm{~cm}$, were obtained from commercial suppliers. Animals were placed in crushed ice for at least $1 \mathrm{hr}$ prior to surgery and all surgical procedures were performed under hypothermic conditions.

A dorsal laminectomy to expose the second to fifth dorsal spinal segments was performed (for details, see Lennard and Stein, 1977). In two animals, the hindlimb enlargement of the spinal cord was bilaterally deafferented. This was accomplished by performing a second dorsal laminectomy extending from the eighth dorsal to the first sacral spinal segment. The dorsal roots of the eighth through tenth dorsal spinal segments and the first sacral spinal segment were then sectioned. In all animals, two nerve-cuffs were implanted in the hindlimb to be examined (Fig. 1C). One nerve-cuff was placed on the triceps femoris, pars ambiens muscle-nerve (CUF-MN) near the point of entrance into the muscle. A second nerve-cuff was positioned on a cutaneous nerve branch (CUF.CUT), which courses through ambiens, at a point distal to its exit from the muscle. Each nerve-cuff consisted of a 3- to 4-mm length of polyethylene tubing (inside diameter, $0.38,0.58$, or $0.76 \mathrm{~mm}$ ) with the bared ends of two $100-\mu \mathrm{m}$ enameled silver wires affixed to the inside surfacc of the tubing (typical interelectrode distance of about $2 \mathrm{~mm}$ ). The enameled leads of the two electrodes were glued together with cyanoacrylate cement. The leads of each nerve-cuff were routed subcutaneously along the leg and out through a small hole drilled in the ipsilateral fourth costal scute of the carapace.

Bipolar EMG electrodes were implanted in tive to seven muscles of the hindlimb to be examined (Fig. 1). In all cases, EMG leads were placed in the following three muscles: triceps femoris, pars ambiens (AMB)-a knee extensor, femoral adductor; triceps femoris, pars femorotibialis (FEM TIB)a single joint knee extensor; and flexor cruris, pars flexor tibialis internus (FLEX TI) - a hip retractor, knee flexor. Other muscles which received EMG leads included: iliofemoralis, anterior head (ILFEM) - a hip protractor, femoral abductor; iliofibularis - a hip retractor, knee flexor, femoral abductor; extensor digitorum communis - a dorsiflexor of the foot; and gastrocnemius, femoral head-a plantar flexor of the foot which assists in web spreading. Details of muscle nomenclature and anatomy can be obtained from the monograph by Walker (1973).

All EMG electrodes consisted of two $100-\mu \mathrm{m}$-diameter enameled silver wires glued together with cyanoacrylate cement. The two leads of the bipolar electrode were stripped of enamel at one end and soldered to two of the 12 pins of a FR-12S microminiature receptacle (Microtech inc., Boothwyn, PA).

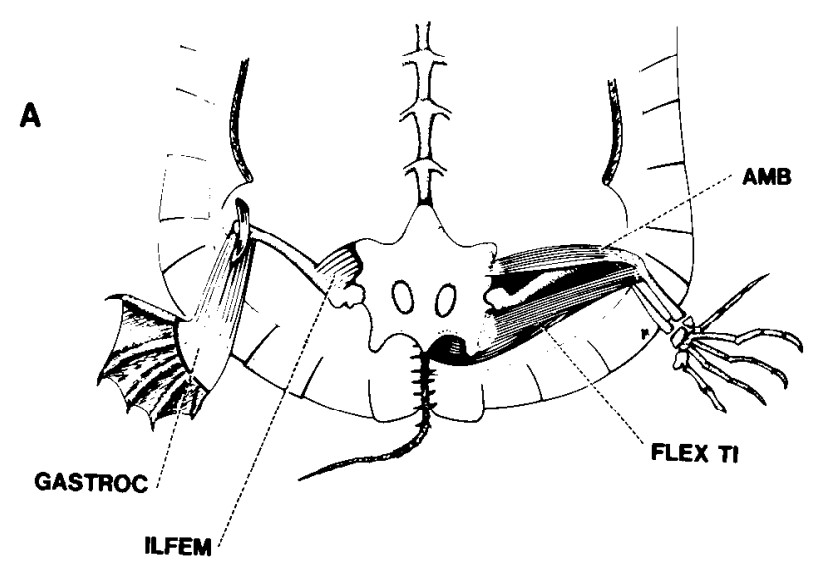

B

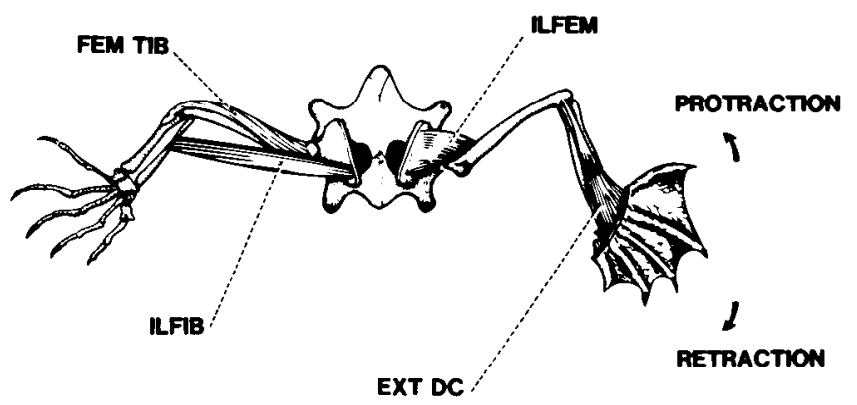

C

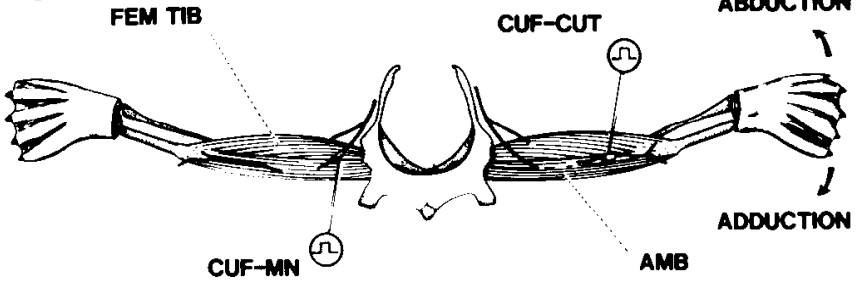

Figure 1. Anatomical sketch indicating the muscles from which EMG recordings were obtained and the position of stimulating nerve-cuffs. EMG and nerve-cuff implants. were made within a single hindlimb, but have been depicted bilaterally for graphic clarity. Only muscles utilized in the study have been represented. $A, A$ ventral view with the plastron and the flexor cruris muscle group of the right hindlimb removed. $B$, A dorsal view of the pelvis and hindlimbs. Arrows in $B$ indicate the direction of the powerstroke (retraction) and returnstroke (protraction) for both the dorsal and ventral views. $C$, A cranial viow of the pelvis and the hindlimbs showing the nerve innervating $\mathrm{AMB}$ and its distal continuation as a cutaneous nerve branch. A stimulating nerve-cuff (CUF-MN) is shown encircling the AMB muscle-nerve of the right hindlimb ( $(\mathrm{e} / \mathrm{t}$ side of $\mathrm{C}$ ) prior to the nerve's entry into the muscle. A stimulating cutaneous nerve-cuff (CUF-CUT) is shown encircling the cutaneous branch of the nerve following the distal exit of the nerve from the AMB muscle of the left hindlimb (right side of $C$ ). Arrows in $C$ indicate the plane of abduction and adduction. Muscles depicted include: triceps femoris, pars ambiens $(A M B)$; triceps femoris, pars femorotibialis $(F E M$ TIB); flexor cruris, pars flexor tibialis internus (FLEX TI); iliofemoralis (ILFEM); iliofibularis (ILFIB); extensor digitorum communis (EXT DC); and gastrocnemius (GASTROC). 

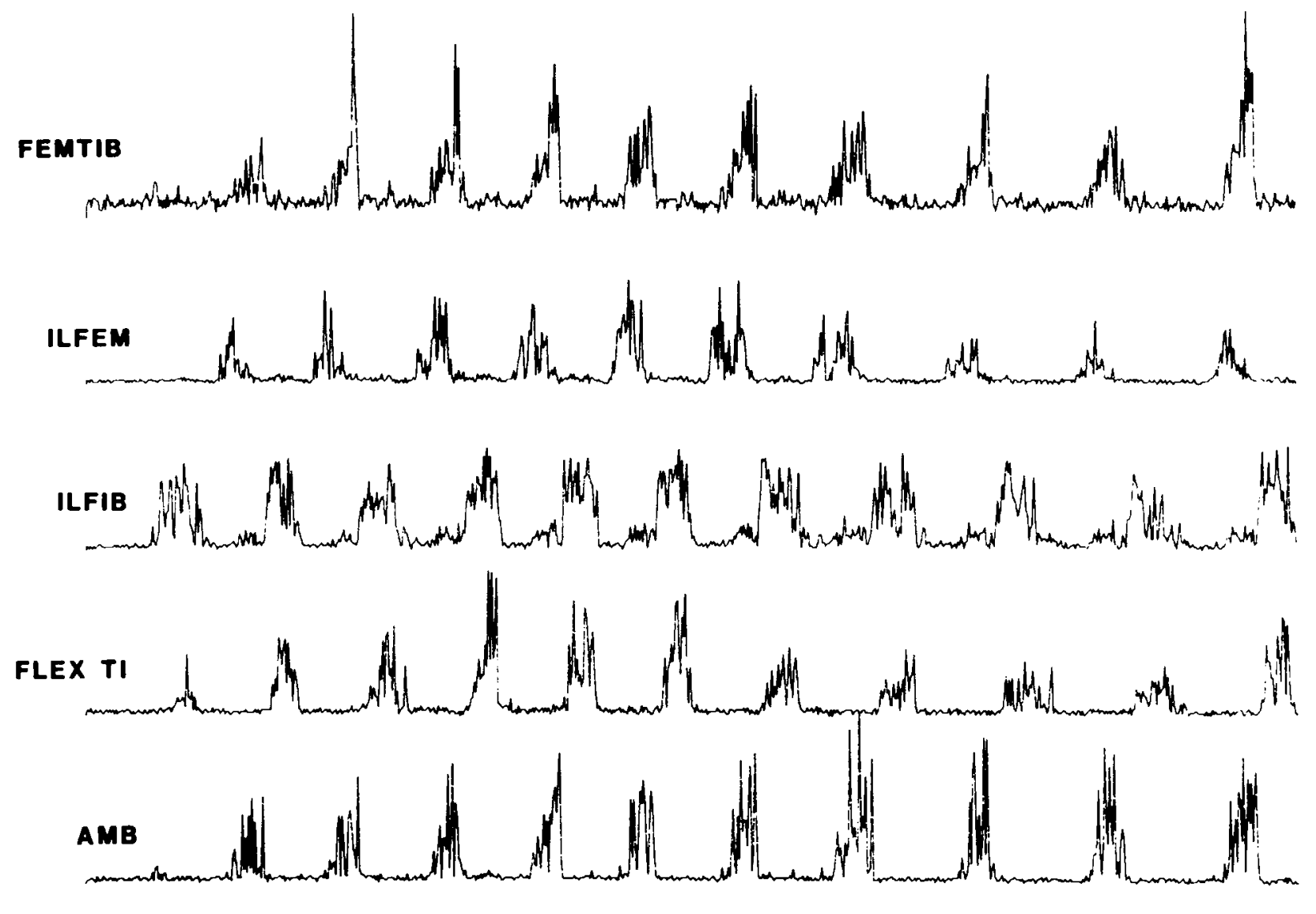

Figure 2. Rectified and integrated EMG pattern from five muscles during electrically elicited swimming. The last quarter of the 10-sec swimming sequence is not shown. There is EMG activity in the hip retractor-knee flexors, FLEX T/ and /LFIB, during the powerstroke (limb retracting). The onset of EMG activity in the hip protractor-femoral abductor ILFEM preceeds the activity in the knee extensors AMB and FEMTIB. The returnstroke consists of an early hip protraction phase followed by knee extension prior to the onset of the next powerstroke (see Lennard and Stein, 1977 for kinematic details). The time bar is $1 \mathrm{sec}$.

The other end of the electrode was "flush" cut to provide a highly selective EMG electrode (recording surface consisting of two $100-\mu \mathrm{m}$ cross-sectional areas separated by the thickness of the enameling). One or two receptacles, each capable of interfacing up to six EMG electrodes, were placed in lucite hoiders glued to the external surface of the turtle's carapace. Skin incisions were made above each of the muscles to be implanted. The electrodes were inserted through the carapace hole used for the nerve-cuff leads, threaded subcutaneously to the appropriate incision, and then implanted into the body of the muscle. Skin incisions were sutured and the carapace hole was plugged with bone wax. The animal was allowed to warm to room temperature following all surgical procedures. Recording sessions began the next day and extended over a 2- to 3-day period.

\section{Recording and stimulating protocols}

For recording, the turtle was partially immersed in water by means of a band clamp which encircled the body at the level of the shell bridge. EMG electrodes were connected to AC preamplifiers by means of Microtech FP$12 S$ microminiature plugs and MS-12 twelve-conductor shielded cabling Swimming sequences expressed in only one hindlimb (Lennard and Stein, 1977) were elicited via a 10-sec duration constant frequency $(20$ to $80 \mathrm{~Hz}$ ) electrical stimulation (pulse duration, $0.1 \mathrm{msec}$; pulse amplitude, 10 to $15 \mathrm{~V}$ ) delivered to the contralateral dorsolateral funiculus (DLF) of the third or fourth segment of the spinal cord. The stimulus was delivered through a manipulatormounted concentric needle electrode (MCE-100; Rhodes, Woodland Hills, CA). An intertrial interval of 2 min separated each 10-sec swimming sequence. Midway through most sequences, a single DC-coupled 0.1-to 0.3msec pulse from a constant-voltage optical isolator was delivered through the nerve-cuff on either the AMB muscle-nerve or the cutaneous nerve. Every third or fourth swimming sequence served as a control sequence, during which no nerve-cuff stimulus was applied. During each swimming sequence, raw EMG data and monitors of the DLF and nerve-cuff stimuli were digitized on a laboratory computer system. All EMG recordings and the DLF stimulus monitor were sampled at $1 \mathrm{KHz}$. The precise time of delivery of the nervecuff stimulus was represented as a single input to an event detector (Schmitt trigger). In some experiments, information was recorded on an instrument tape recorder for later conversion.

Parameters for nerve-cuff stimulation were set at the beginning of each day's experiment and reconfirmed several times during the course of the experiment. Stimulus parameters were always determined during periods when the animal was not displaying spontaneous limb movements. Voltage and pulse duration for $\mathrm{AMB}$ muscle-nerve stimulation was set slightly above threshold (typically less than $1.5 \mathrm{~T}$ ) and had to meet the following criteria: (1) a short latency ( 2 to $4 \mathrm{msec}$ ) AMB EMG response which lasted 10 to 15 msec; and (2) no EMG response in any other muscle for single stimulus trials or when 10 consecutive trials were signal averaged. The near-threshold stimulus did result in occasional response failures during swimming. During data analysis, swimming sequences were screened for failures. If the CUF. $M N$ stimulus arrived during a period of $A M B$ inactivity and failed to elicit an EMG response, the swim sequence was discarded. Stimuli delivered during periods of AMB EMG activity could not be effectively screened, since the lack of response was obscured by ongoing muscle activity. Cutaneous nerve-cuff stimulation levels were typically in the range of 2 to 3 times thrcshold for an EMG rcsponse in ILFEM. Two criteria were used for setting the CUF-CUT stimulus voltage: (1) the stimulus had to elicit an observable flexion reflex response of the hindlimb when the animal was not swimming; and (2) during DLF-elicited swirnirning sequences, the CUF-CUT stimulus did not cause hindlimb withdrawal or the cessation of locomotor activity.

\section{Data analysis}

Following an experiment, the digitized raw EMG data and DLF stimulus monitor from each swim sequence were digitally rectified and integrated with a $10-\mathrm{msec}$ time constant. In Figure 2, the rectified and integrated EMG 


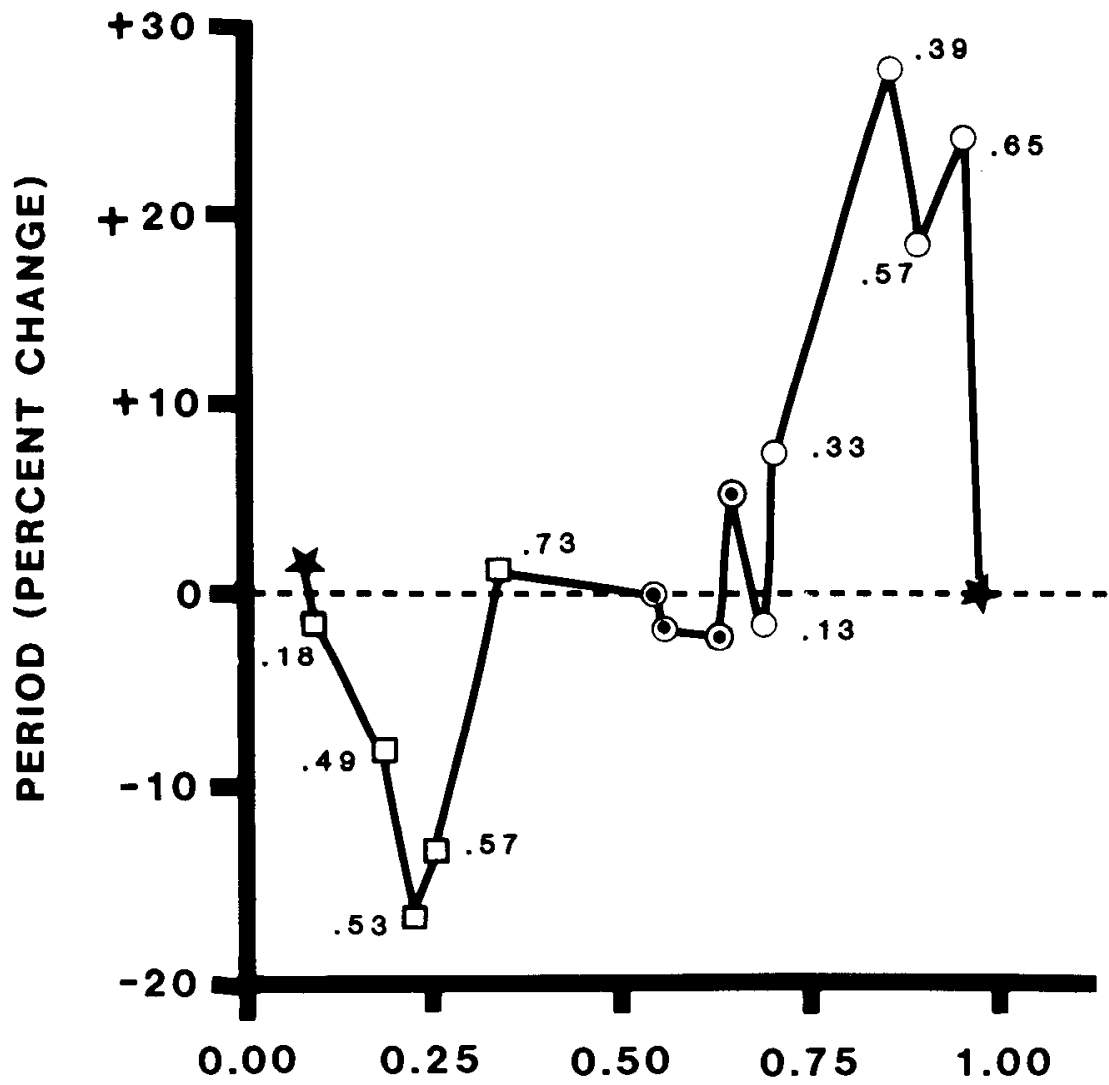

Figure 3. Changes in the period of the Nth swim cycle plotted as a function of the phase of CUF-MN stimulus delivery. Period changes are represented in terms of a percentage increase (plus values) or decrease (minus values) from a matched control swim cycle period. The phase of stimulus delivery within the swim cycle has been normalized by dividing stimulus latency by the period of a matched control swim cycle. Squares and circles are used to indicate stimulus delivery during EMG activity in FLEX $\mathrm{TI}$ and $\mathrm{AMB}$, respectively. In addition to phase values, stimulus delivery is represented as a time corresponding to some fraction of the mean prestimulus EMG burst duration in FLEX TI (numbers adjacent to squares) or AMB (numbers adjacent to circles). Bullseyes indicate stimuli delivered after the termination of FLEX TI EMG activity but prior to the onset of activity in AMB. Stars represent stimuli falling at the transition from the end of the Nth cycle to the onset of the Nth+1 cycle. All swimming sequences are from the same turtle.

\section{PHASE}

TABLE I

Phase-dependent changes resulting from muscle-nerve stimulation

A nonparametric analysis was employed to determine if the effects of CUF-MN stimuli delivered at different times within the Nth swim cycle were statistically significant. FLEX TI and AMB were used as examples of powerstroke and returnstroke muscles, respectively. Statistically significant increases or decreases in swim cycle period, EMG burst duration, and interburst interval are indicated along with the calculated probability value. All data are from one turtle. Four swimming sequences are used for the SPD and SPA groups, and six sequences are used for the ISP group. No statistically significant changes were found when the Nth+1 cycle of the same swimming sequences was tested.

\begin{tabular}{|c|c|c|c|c|c|}
\hline \multirow{2}{*}{ Stimulus Delivery Time } & \multirow{2}{*}{ Swim Cycle Period } & \multicolumn{2}{|c|}{ AMB } & \multicolumn{2}{|c|}{ FLEX TI } \\
\hline & & $\mathrm{BD}^{\mathrm{a}}$ & $\mathrm{IBI}$ & $\mathrm{BD}$ & $|\mathrm{B}|$ \\
\hline SPD & $\mathrm{I}(p<0.01)$ & $1(p<0.03)$ & $*^{b}$ & * & $1(p<0.002)$ \\
\hline SPA & $\mathrm{D}(p<0.03)$ & * & $\mathrm{D}(p<0.01)$ & $\mathrm{D}(p<0.01)$ & * \\
\hline
\end{tabular}

${ }^{a} B D$, EMG burst duration; $I B \mid$, interburst interval; $I$, statistically significant increases; $D$, statistically significant decreases in swim cycle period.

${ }^{\circ} *$, no statistically significant change $(p<0.05)$.

pattern seen during swimming is shown for a control swim sequence elicited by DLF stimulation. The EMG and stimulus data were then displayed on a graphics terminal where an operator-driven cursor was used to obtain values for the onset and termination of each EMG burst, as well as the time of arrival of the nerve-cuff stimulus. Graphic resolution was $10 \mathrm{msec}$, and operator repeatability of choice of burst onset and termination point was typically \pm 20 msec.

The production of a single swimming stroke requires the orderly activation of each hindlimb muscle for an appropriate time during the locomotor cycle. In order to quantify this intracycle motor pattern, a number of different values were calculated on a cycle-by-cycle basis, using each recorded muscle in turn as the referrent. Cycle period was measured from the onset of EMG activity in a muscle to the beginning of the subsequent EMG burst in that muscle. EMG burst duration, defined as the time from the onset to the termination of EMG activity, and interburst interval, defined as cycle period minus burst duration, were also determined. Latency, the time from the beginning of the cycle in the referrent muscle to the onset of EMG activity in each of the other muscles, was obtained in order to derive phase values for EMG activity in each muscle with respect to the referrent muscle. Phase was calculated by dividing latency by the cycle period of the referrent muscle. To further quantify the intracycle motor pattern, all measures were also calculated using EMG burst terminations to define cycle period. Means and SDs for each measure were obtained for cycles preceding the nerve-cuff stimulation (all cycles were averaged for control swimming sequences without nerve-cuff stimulation). EMG amplitudes were not quantified in the present study.

Several methods were employed to assess the temporal effects of nerve stimulation on the intracycle motor pattern of the locomotor cycle which was in progress at the time of stimulus delivery. This cycle will be referred to as the Nth cycle or the perturbed cycle. The EMG phase relationships among muscles in the Nth cycle were determined and then compared to those obtained in prior and subsequent cycles of that swimming sequence. The effects of peripheral nerve stimulation on cycle period were also examined. For this analysis, the cycle period of the major powerstroke muscle, FLEX $\mathrm{TI}$, was used as a measure of the overall swimming period and is referred to in the text as the swim cycle period. The Nth swim cycle period of swim sequences in which a nerve was stimulated was compared to the corresponding swim cycle period of the temporally closest control (no nerve 


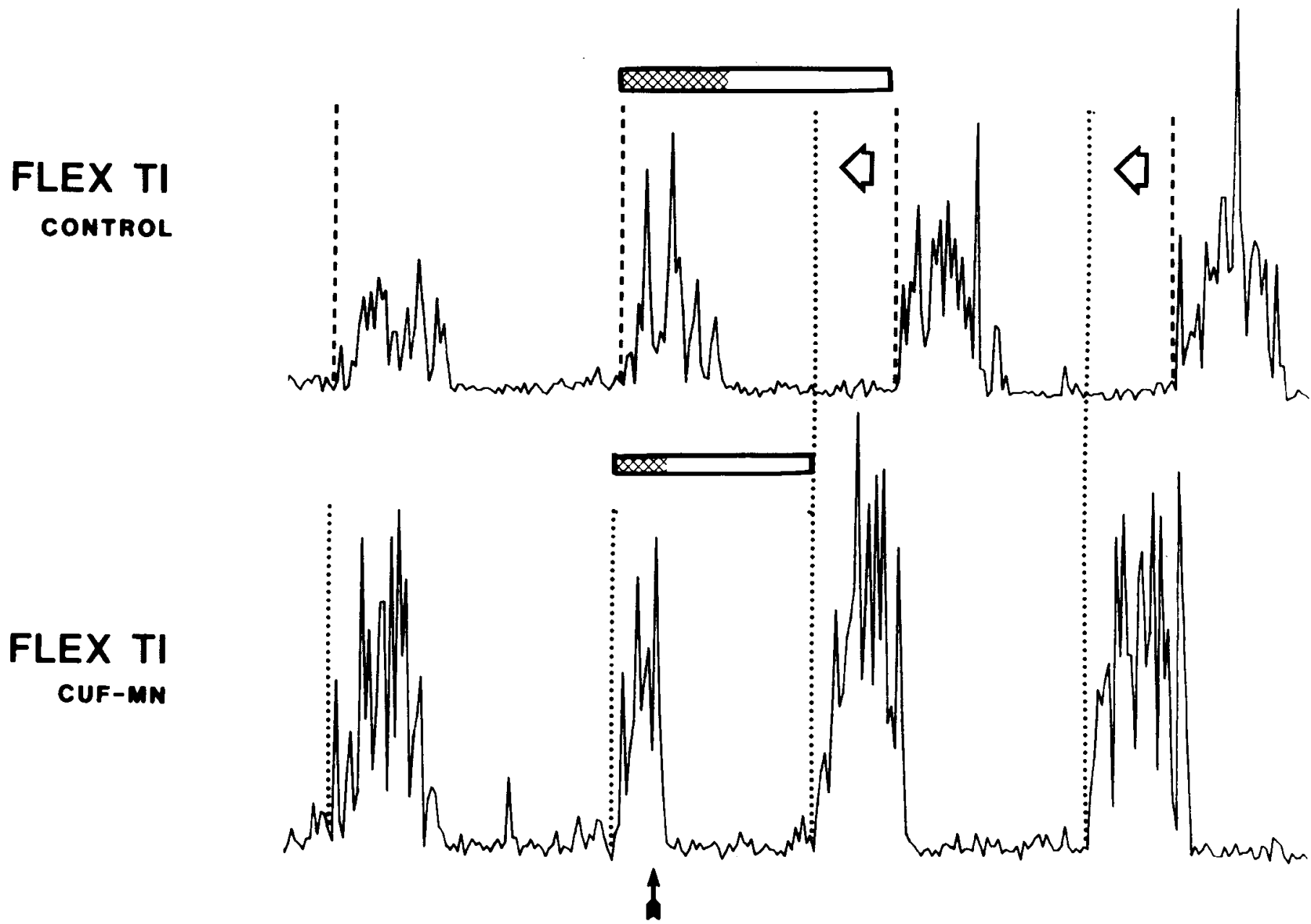

Figure 4. Changes in FLEX TI EMG activity resulting from CUF-MN stimulation during the SPA. The bottom trace shows EMG activity in FLEX TI during the CUF-MN perturbed cycle (Nth cycle) and the previous (Nth-1) and subsequent (Nth+1) swim cycles of an experimental swimming sequence. Stimulus delivery (arrow) was in the fifth cycle of the swimming sequence. The top trace shows FLEX TI EMG activity during the fourth through sixth swim cycles of a control swimming sequence (no CUF-MN stimulus) elicited $2 \mathrm{~min}$ earlier. The onsets of the fourth cycle from each swimming sequence have been aligned. Cycle onsets are indicated by dashed lines and dotted lines for the control and experimental sequences, respectively. CUF-MN stimulation shortens the Nth swim cycle period (lower bar) by an in-phase decrease of the FLEX TI EMG burst duration (shaded portion of lower bar). These effects can be seen by comparing the Nth cycle period and burst duration with the corresponding control values (upper bar). The decrease in the Nth cycle swim period advances (open arrow head on the left) the onset of the next cycle ( $\mathrm{Nth}+1$, dotted line) to a time earlier than predicted by the control swimming sequence (sixth cycle onset, dashed line). Since the period of the Nth+1 swim cycle is not altered by CUF-MN stimulation, the phase shift of the Nth +1 cycle is maintained, and there is an advance in the onset of the $\mathrm{Nth}+2$ swim cycle (open arrow head on the right).

stimulus) swimming sequences. The time of stimulus delivery within the Nth cycle was expressed in terms of the phase of the stimulus with respect to the swim cycle. To provide a normalized time of stimulus delivery, which was not subject to effects of the stimulus on the period of the Nth cycle, phase values were computed as the latency from the beginning of the perturbed swim cycle to the time of stimulus delivery, divided by a swim cycle period value from the corresponding cycle of the temporally closest control swimming sequence. To assure a valid match between stimulated and control swimming sequences, only sequences in which the period of the cycle immediately preceding the Nth cycle was within $5 \%$ of the period of the corresponding control swim cycle were used in data analysis. The stimulus was also represented as falling at a time corresponding to some fraction of the projected total EMG activity of AMB or FLEX TI during the perturbed cycle. This value was calculated by taking the latency from the burst onset to the stimulus and dividing by the average burst duration for the swim cycles prior to the perturbed cycle.

Nonparametric statistics were used to evaluate the effects of stimuli delivered at different times during the perturbed swim cycle. Preliminary data analysis indicated that norvo cuff stimuli produced noticeable alterations in the Nth cycle when stimulus arrival time fell midway during either the AMB or FLEX TI EMG burst (e.g., see Fig. 3). In order to verify and quantify these observations, all swimming sequences were assigned to one of three groups: (1) The first group consisted of all swimming sequences in which the stimulus was delivered during the middle third of the FLEX TI burst; (2) all sequences in which the stimulus was delivered during the middle third of the AMB burst were assigned to a second group; and (3) a third group was employed for all other stimulus delivery times. Period, burst duration, and interburst interval were tested using a nonparametric rank-sum procedure which separately ranked, from 1 (low) to 7 (high), each of the variables for seven cycles including: the Nth cycle; the three prior cycles (Nth-1 to Nth-3); and the three subsequent cycles $(\mathrm{Nth}+1$ to $\mathrm{Nth}+3)$. The Nth cycle rank for all swimming sequences of a single group were summed together. Tables, based on the number of swimming sequences in a group and all possible rank orders of the seven cycles of each sequence, were used to determine the probability that the rank sum of the Nth cycles would have a value as small or smaller than the value obtained. Probabilities less than 0.05 indicated a statistically significant decrease in the parameter of the Nth cycle being tested. The probability of a rank sum as great or greater than the Nth cycle rank sum obtained was then determined. In this case, probabilities of less than 0.05 indicated a statistically significant increase in that parameter of the Nth cycle. The entire test was then repeated for the cycle following the perturbed cycle by using the Nth+1 rank sum values.

A swimming sequence consists of multiple locomotor cycles. The temporal pattern of this repeating series of locomotor cycles can be thought of as the swimming rhythm. In addition to assessing the effects of nerve stimulation on the Nth cycle, changes in the overall timing of the poststimulus swimming 

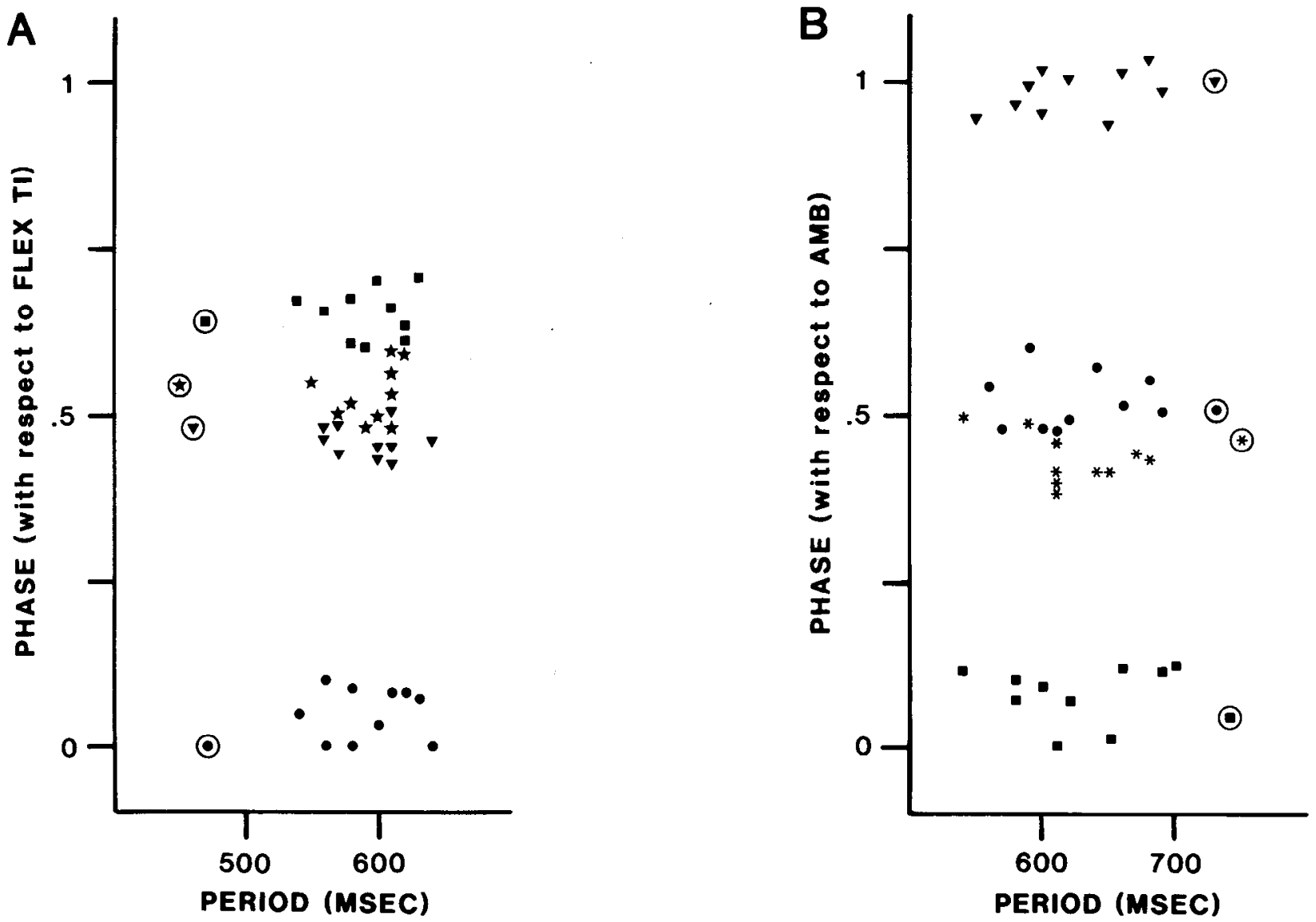

Figure 5. Normal intralimb phase relationships among muscles are preserved during period changes resulting from CUF-MN stimulation. A, The phase of four muscles with respect to the onset of FLEX TIEMG activity is plotted against cycle period for each muscle during a single swimming sequence in which a CUF-MN stimulus during the SPA shortened the perturbed cycle. $B$. The phase of four muscles with respect to the onset of AMB EMG burst activity is plotted against cycle period for each muscle during a single swimming sequence in which a CUF-MN stimulus during the SPD lengthened the perturbed cycle. In $A$ and $B$, phase and period values for the perturbed cycle are represented by encircled symbols. While CUF-MN stimulation has shifted period values for the perturbed cycle, the phase relationships between muscles are unchanged and fall within the range of phases seen during preceding and subsequent unperturbed cycles. The specific muscles depicted include: gastrocnemius $(\boldsymbol{\theta})$, extensor digitorum communis $(\boldsymbol{\nabla}), \mathrm{AMB}(\star), \mathrm{FEM}$ TIB $(\boldsymbol{D})$, and FLEX TI (*). In $A$ and $B$, the first 11 cycles of the swimming sequence are shown. Stimulus delivery was during the sixth cycle in $A$ and the fifth cycle in $B$.

rhythm were examined. In order to quantify poststimulus alterations in the swimming rhythm, experimental swimming sequences, in which a stimulus was delivered, were matched to a nonstimulated control sequence. The phase of onset of each swim cycle (onset defined as the beginning of FLEX TI EMG activity) in the experimental swim was then calculated with respect to the cycle onset expected if no stimulus had been delivered. The phase measure was obtained by first assigning a time value of zero to both the onset of the swim cycle in which the stimulus arrived and to the corresponding cycle of the control sequence (in some cases, a zero time value was assigned to the third swim cycle for both the experimental and control swim). The timing of the subsequent cycles was then determined by a cumulative addition of measured swim cycle periods for each swim to the zero values. The phase of the experimental cycle onset, with respect to the expected onset time, could then be calculated by subtracting control from experimental onset time and dividing the difference by the control swim cycle period Alterations in the swimming rhythms were typically examined in 4 to 7 poststimulus cycles, the exact number depending on the time of stimulus delivery within the 10-sec swimming sequence.

\section{Results}

During a swimming sequence, the period of the ongoing swim cycle could be perturbed by delivering a single electrical pulse to the ambiens muscle-nerve (CUF-MN). The specific effect of CUFMN stimulation on swim cycle period was found to be phasedependent, that is, to be related to the time of stimulus delivery within the swim cycle (Fig. 3). The period of the swim cycle in progress at the time of stimulus delivery (Nth cycle) only differed from control values if the CUF-MN stimulus occurred during one of two specific parts of the swim cycle. These two parts of the swim cycle can be viewed as time windows during which CUF-MN stimulation can alter the locomotor output. The first of these windows was about a quarter of the way through the swim cycle, during the swirnming powerstroke when there was EMG activity in the hip retractor/knee flexor muscle, FLEX TI. If CUF-MN stimulation occurred at phase values centered around 0.25 , the period of the Nth swim cycle was decreased (Fig. 3). Stimulus delivery can also be expressed as happening at a time which corresponds to a percentage of the lotal EMG burst duration of FLEX TI. The window in which CUF-MN stimulation decreased the period of the Nth swim cycle spanned the middle third of the FLEX TI EMG burst (Fig. 3, values associated with squares indicate stimulus times calculated as a percentage of FLEX TI burst duration). Shortening the Nth swim cycle period advances, to an earlier than expected time, the onset of the subsequent $(\mathrm{Nth}+1)$ swim cycle. Therefore, the time window centered around phase value of 0.25 and spanning the middle third of the FLEX TI EMG burst, has been designated as a sensitive period for advancing (SPA) the onset of the Nth+1 swim cycle.

A second time window in which CUF-MN stimuli perturbed the locomotor output occurred late in the swim cycle at phase values centered around 0.85 and spanning the middle third of the AMB EMG burst (Fig. 3 , circles). The period of the Nth swim cycle was increased by CUF-MN stimuli delivered within this time window, which thus represents a sensitive period for delaying (SPD) the $\mathrm{Nth}+1$ swim cycle. The SPD corresponded to a time late in the 
Figure 6. Alterations of the swimming rhythm resulting from stimulation during the SPA. In each of the swimming sequences, stimulus delivery shortened the $N$ th swirr cycle and advanced (negalive $\phi_{\text {perturbed, expected }}$ values) the onset of the Nth +1 swim cycle (PostStimulus Cycle \#1). A, During two of the swimming sequences (solid circles) a CUF-CUT stimulus resulted in a temporary phase shift of the locomotor rhythm (phase values return to zero). During a third swimming sequence from the same experiment, CUF-MN stimulation produced a permanent phase shift of the locomotor rhythm (phase values do not return to zero). $B$, The permanent phase shift of the locomotor rhythm is due to a change in the period of only the Nth swim cycle. In the bottom panel, swim cycle period is plotted for both an experimental swimming sequence (solid circles) during which a CUF-MN stimulus was delivered and the control swimming sequence (open circles) used as a referrent to determine $\phi_{\text {perturbed, expected. In the }}$ top panel, phase values near zero indicate the close match between control and experimental swimming sequence rhythms prior to CUF-MN stimulus delivery (double-headed arrow) during cycle \#7 (Nth swim cycle). The experimental swimming sequence in $B$ is also shown in $A$ (open circles).

A
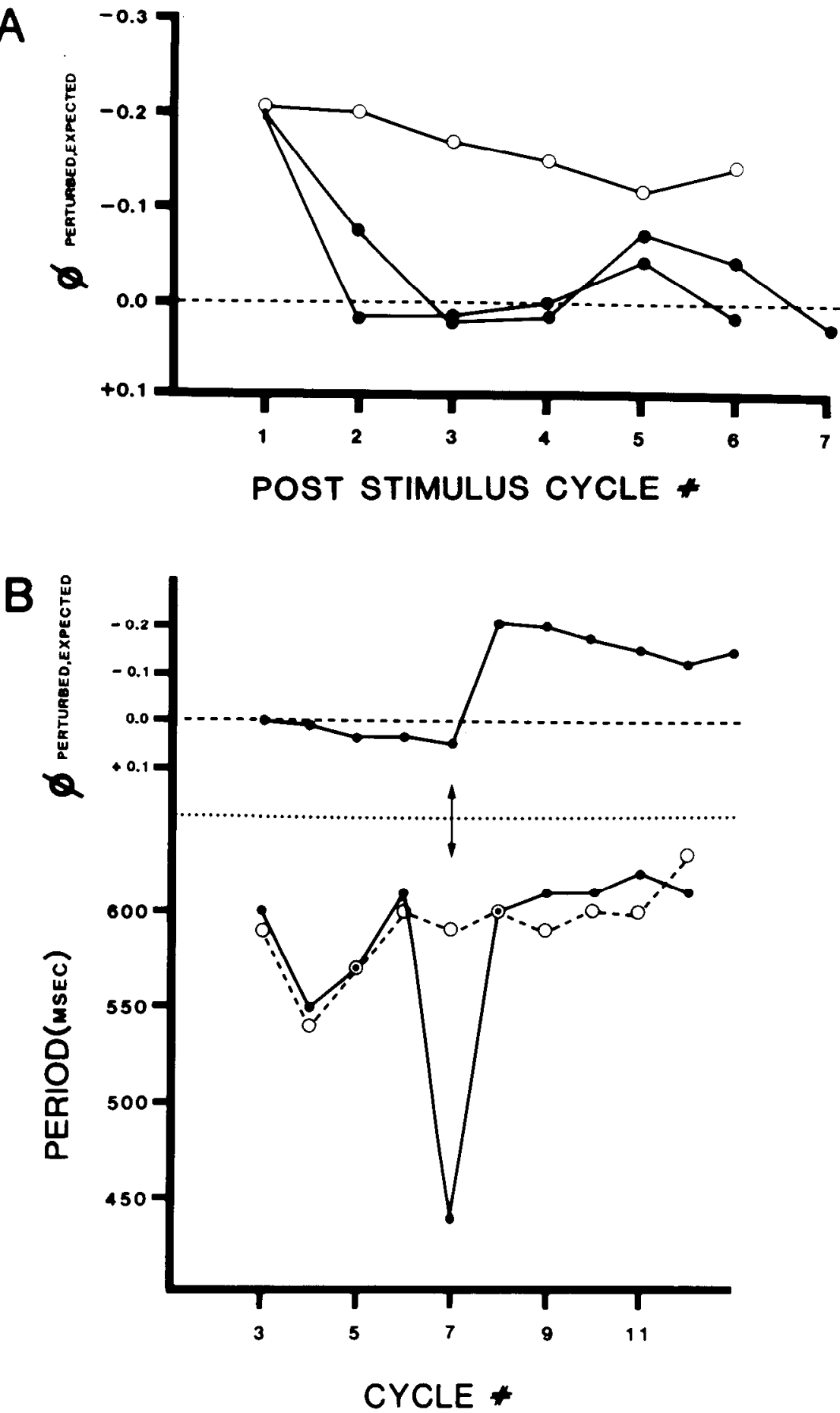

swimming returnstroke, following hip protraction and during knee extension (see Lennard and Stein, 1977 for kinematic details). The SPA and SPD represented the only times during the swim cycle when period was effected by CUF-MN stimulation. These sensitive periods were separated by two insensitive periods (ISP) which encompassed the rest of the swim cycle. In two animals, CUF-MN stimulation was tested following bilateral dorsal rhizotomies of the hindlimb enlargement. These experiments were employed to control for the possibility that CUF-MN-induced period changes were due to antidromic activation of motor axons. In both deafferented animals, no time within the swim cycle was found during which CUF-MN stimuli could alter swim cycle period

Single stimuli delivered to the ambiens muscle-nerve during the sensitive periods produced in-phase adjustments of muscle activity.
That is, if a muscle was active at the time of stimulus delivery, the EMG burst duration would change. But, if a muscle was not displaying EMG activity, stimulus delivery would result in a change in the ongoing interburst interval. Table I summarizes a nonparametric statistical analysis of the in-phase changes which resulted from CUFMN stimulation. Stimulation during the SPD produced a statistically significant increase in the period of the Nth swim cycle. The longer swim cycle period in response to SPD stimulation was paralleled by a significant increase in the ongoing AMB EMG activity and by a prolongation of the FLEX TI interburst interval in progress at the time of stimulus delivery. CUF-MN stimulation during the SPA significantly decreased the period of the perturbed cycle. This Nth cycle period decrease was accompanied by a shortening of the ongoing interburst interval in $\mathrm{AMB}$, without a change in duration of the subsequent 


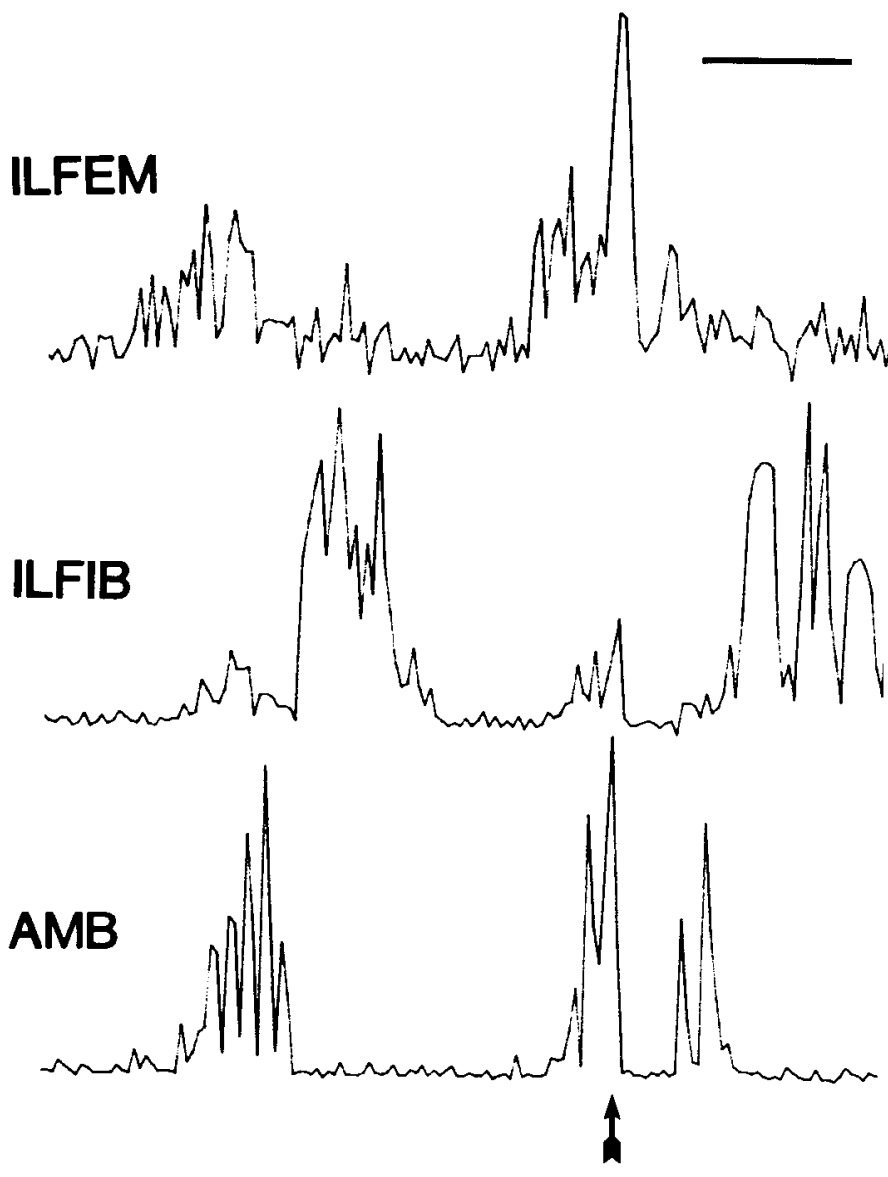

Figure 7. EMG flexion reflex response embedded within a period increase resulting from CUF-CUT stimulation during SPD. Following stimulation (arrow), there is excitation of EMG activity in musclos participating in the flexion reflex (ILFEM) and a decrease of ongoing EMG activity in muscles which are normally inhibited during the flexion reflex ( $A M B$ and low-level early component of $/ L F(B)$. The time bar is $250 \mathrm{~m}$ isec

AMB EMG burst. Consistent with CUF-MN stimulation altering only EMG activity and interburst intervals in progress at the time of stimulus delivery, SPA stimulation produced a shorter duration EMG burst in FLEX TI, but did not alter the subsequent interburst interval. Figure 4 illustrates the alteration of FLEX TI EMG activity resulting from CUF-MN stimulation during the SPA. The shortening of the Nth swim cycle and the concomitant early onset of the Nth+1 cycle clearly reflect a decrease in the duration of the FLEX TI EMG burst. In contrast to CUF-MN stimulation during the SPA or SPD, stimulus delivery during either of the two insensitive periods did not produce statistically significant changes in any of the parameters of the Nth cycle (Table I).

The swimming stroke reflects an underlying intracycle motor pattern, i.e., an orderly activation of each limb muscle for the appropriate time during the locomotor cycle. The phase relationships between muscles were examined to see if there were alterations in the timing between muscles when CUF-MN stimulation during the sensitive periods resulted in a perturbed cycle. If the phase relationship between a pair of muscles was maintained, then the stimulation did not alter the timing relationship between the two muscles. If the timing relationship between each pairwise combination of muscles remained unchanged by CUF-MN stimulation, then the intracycle motor pattern was not perturbed. Figure 5 depicts phase maintenance during both SPA (Fig. 5A) and SPD (Fig. 5B) CUF-MN stimulation. Stimuli which altered the period of the perturbed cycle (encircled symbols) also resulted in a change in the latency between the pair of muscles. The net effect of these latency changes was to maintain the normal phase relationship between muscles in spite of changes in the period of the perturbed cycle. CUF-MN stimulation during the sensitive periods was found to produce in-phase adjustments in either the burst duration or interburst interval of each muscle, such that the phase relationships between all pairs of muscles examined were maintained, and the normal intracycle motor pattern was expressed.

Cutaneous nerve stimulation delivered during the sensitive periods produced changes in the ongoing swim cycle which were similar to the perturbations resulting from AMB muscle-nerve stimulation (Fig. $6 A$ ). CUF-CUT stimulation during the sensitive periods, like musclenerve stimulation, did not significantly perturb the intracycle motor pattern even though there were changes in cycle period and alterations of EMG burst durations and interburst intervals.

In contrast to the SPD for muscle nerve stimulation, the SPD for cutaneous stimulation extended over a greater portion of the swim cycle, encompassing the entire returnstroke. There were no major changes in the duration or time of appearance of the SPA. In addition to the alterations of the ongoing swim cycle produced by CUF-CUT stimulation, a secondary modulation of EMG activity was observed which is consistent with previously reported turtle flexion reflex responses (see Stein et al., 1982). For example a CUF-CUT stimulation during the SPD increased AMB burst duration in a manner similar to a CUF-MN stimulus, but also caused a midburst inhibition of the EMG in AMB (Fig. 7). In summary, the effects of both CUFCUT and CUF-MN stimuli arriving during the sensitive periods were to alter the length of the ongoing state of each musle (i.e., active or quiescent) thus changing the period of the perturbed cycle without producing major changes in the overall timing and coordination of the motor pattern within the cycle.

\section{Alterations of the swimming rhythm}

Muscle-nerve and cutaneous nerve stimulation did produce dramatically different alterations in the timing of the poststimulus swimming cycles, i.e., CUF-MN and CUF-CUT had different effects on the onset time for the $\mathrm{Nth}+2$ and all subsequent swim cycles. In Figure $6 A$, the effect on the swimming rhythm of SPA stimulation of CUF-MN (open circles) and CUF-CUT (solid circles) is illustrated for three swim sequences from the same experiment. For both CUFMN and CUF-CUT stimulation, the phase of the first poststimulus cycle had a negative value indicating a shortening of the Nth cycle period and an earlier-than-expected onset of the Nth+1 cycle. In the case of CUF-MN stimulation, the advance in the onset of the Nth+1 cycle coupled with the lack of significant effect on the period of the Nth +1 and subsequent cycles (Fig. 6B) resulted in a permanent phase shift and the arrival of all poststimulus cycles at an earlierthan-expected time. The two CUF-CUT stimulation sequences shown in Figure $6 \mathrm{~A}$ indicate that although cutaneous nerve stimula tion had an effect similar to CUF-MN on the perturbed cycle, CUFCUT stimulation did not lead to a permanent phase shift. There was an advance in the onset of the first poststimulus cycle, but subsequent cycles returned to the expected time of onset (phase near zero). This can be accounted for by a lengthening of poststimulus cycle periods (typically expressed in the first one to three cycles following the cutaneous stimulus). The compensatory effects of poststimulus period changes on CUF-CUT produced phase shifts is depicted in Figure 8. Both phase (Fig. 8A) and cycle period (Fig. $8 B$ ) are plotted for two swimming sequences in which the CUF-CUT was stimulated during the SPD. The stimulus resulted in an increased period for the perturbed cycle and a delayed onset of the $\mathrm{Nth}+1$ cycle (positive phase value for poststimulus cycle 1; Fig. 8A). A reduction in the period of the cycles immediately following the stimulus, including the $\mathrm{Nth}+1$, leads to a return to a phase value near zero and a return to expected cycle onset times. The swim sequence represented by open circles in Figure $8 B$ also illustrates the fact that the demonstration of a normal $\mathrm{Nth}+1$ period is not sufficient to establish a permanent phase shift, and that the compensatory period changes, associated with temporary phase shifts, may occur over any one of several poststimulus cycles. 
Figure 8. Temporary phase shift of the locomotor rhythm resulting from CUF-CUT stimulation. $A, \phi_{\text {perturted, }}$ expected is plotted against poststimulus cycle number for two swimming sequences in which the stimulus was delivered during the SPD. B, swim cycle period versus poststimulus cycle number is shown for the same two swimming sequences. Poststimulus cycle \#0 indicates the cycle during which CUF-CUT stimulation was delivered (Nth swim cycle). The control swim sequence is represented in $B$ by the dotted line without circles.
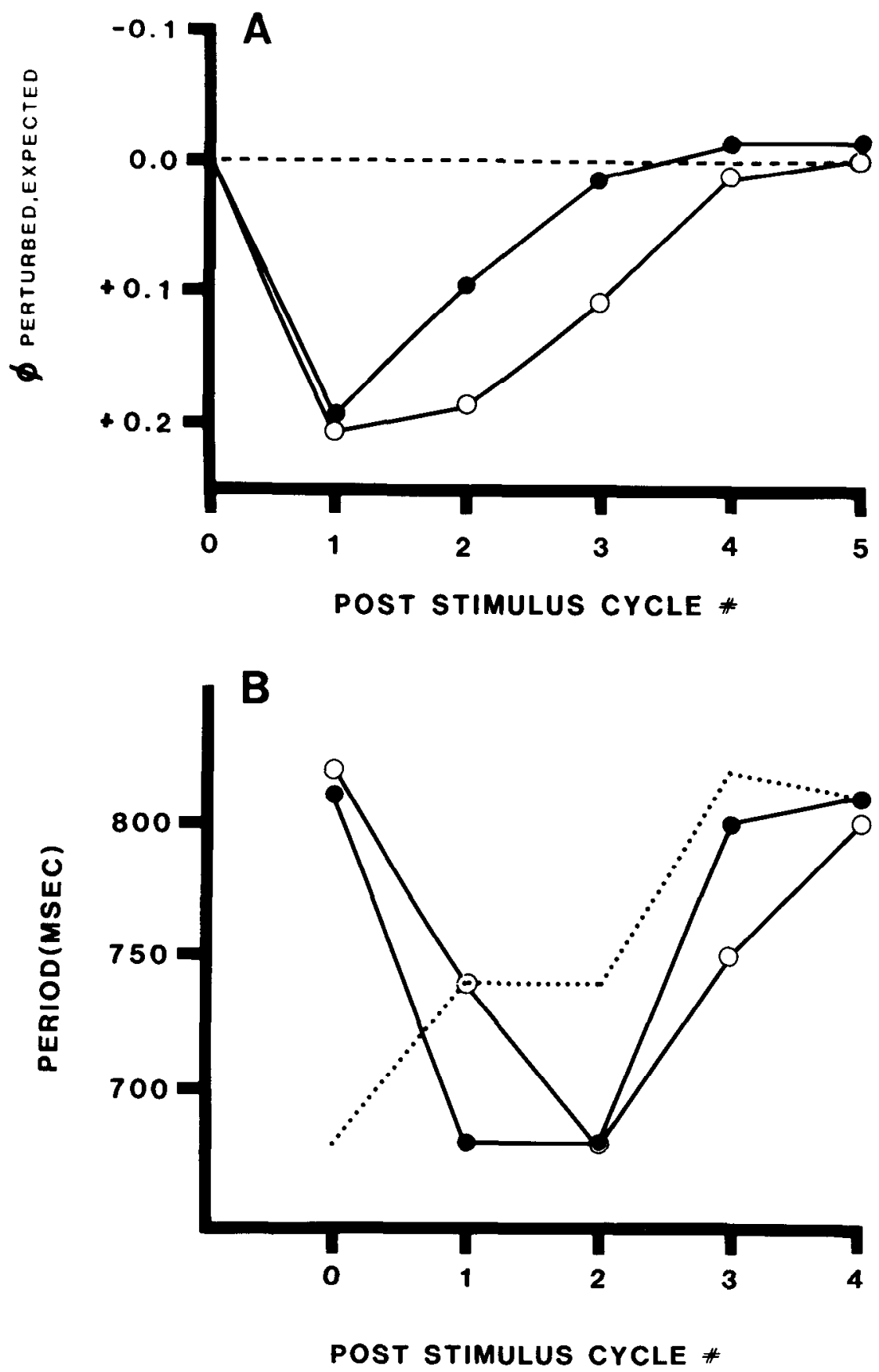

\section{Discussion}

Transient electrical stimulation of an extensor muscle-nerve was found to have a phase-dependent effect on the perturbed locomotor cycle and be capable of permanently resetting the locomotor rhythm for turtle swimming. In a previous study (Duysens and Stein, 1978), muscle-nerve stimulation produced no discernible effect when delivered via nerve-cuffs to cats walking on a treadmill. The contrast of these two results presumably reflects the lack or reduced weighting of tasks other than locomotion, such as postural constraints and crossed hindlimb responses (reviewed in Rossignol et al., 1981) during "monopodal" swimming. In the turtle, stimulation of the muscle-nerve innervating ambiens (a knee extensor and hip adductor) caused a shortening of the perturbed locomotor cycle if the stimulus arrived midway during the swimming powerstroke. This sensitive period (SPA) is at a time when the hip is retracting and the knee joint is extended, but ambiens is not actively contracting. A second sensitive period (SPD) for ambiens muscle-nerve stimulation is coincident with the presumed shortening contraction of ambiens at the end of the returnstroke. Stimulus delivery during the SPD results in a lengthening of the perturbed locomotor cycle.

Muscle spindles are the most likely candidate for the receptor system mediating the phase-dependent effects of muscle-nerve stimulation. The low stimulation strength used for muscle-nerve stimuli would preferentially activate large diameter axons, and is consistent with threshold levels found for single identified turtie spindle afferents (Hermanson and Lennard, unpublished observaseveral intrafusal muscle fibers and a single sensory axon. Only one class of sensory axon, based on diameter and appearance, has been identified. The innervation of the intrafusal fibers is by collaterals of motor axons supplying the extrafusal muscle. The skeletofusimotor innervation of chelonian intrafusal muscle fibers parallels the mammalian $\beta$-system and is typical for reptiles (reviewed by Guthe, 1981). Turtle spindles respond to ramp stretch in a tonic fashion comparable to mammalian spindle secondary endings (Naieje and Crowe, 1974, 1977). The rudimentary tendon organs and joint tions). Crowe and Ragab (1970a, b) found that the turtle spindle has 


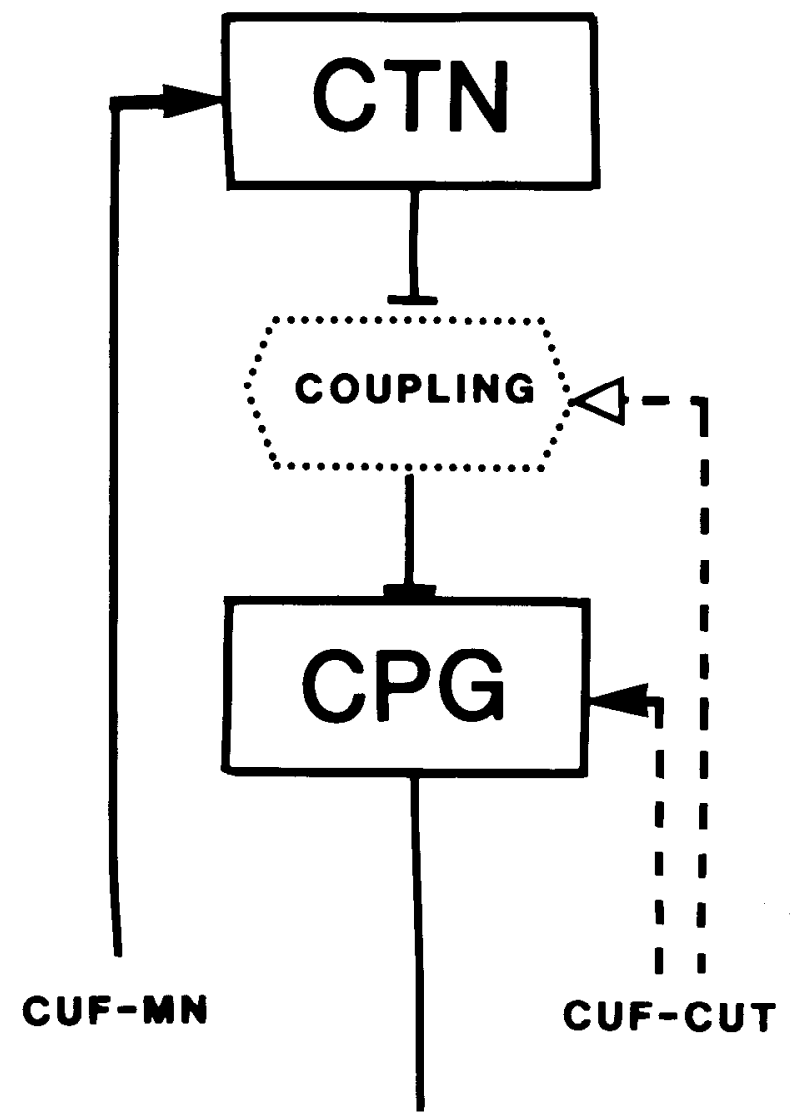

\section{LOCOMOTOR}

\section{OUTPUT}

Figure 9. Hierarchical model of the locomotor network for turtle swimming. The locomotor circuit is separated into an oscillating central timing network (CTN) responsible for producing the locomotor rhythm and a central pattern generator (CPG) which organizes the intracycle motor pattern. Each oscillation of the CTN would drive (___ - $)$ the intracycle CPG to produce the locomotor output for one swim cycle. The CPG may be an oscillator which is entrained by the CTN or a one-cycle pattern generator which is triggered by the CTN. Muscle afferents (solid line, CUF-MN) can access the CTN and permanently phase shift the locornotor rhythm. Cutaneous afferents (dashed lines, CUF-CUT), which can produce a temporary phase shift, reach the CPG and a CNS level which couples (dotted box) the CTN to the CPG. Solid arrowheads indicate a transient increased excitatory influence on central networks during the SPA and a decreased drive during the SPD. The open arrowhead indicates a ramp-like change, which decays over several cycles, in the strength of coupling between the CTN and CPG. CTN/CPG coupling is increased by transient cutaneous input during the SPD and decreased by SPA stimulation

afferents found in turtles (reviewed in During and Miller, 1979) have received little attention and their involvement, if any, in the observed phase-dependent effects is difficult to assess.

The dramatic effects produced by the single-pulse electrical stimulation paradigm were unexpected. It is possible that the central effects of stimulation are due to spatial summation following synchronous activation of many afferents. Alternatively, the phasedependent effects of muscle-nerve stimulation may be derived from a combination of orthodromic activation of afferents and skeletofusimotor efferents. Activation of motor axons could cause a change in either the spindle bias or sensitivity range which may lead to a significant change in the afferent spike train code. The possibility that the reported phase-dependent effects were due exclusively to stimulation of motor axons has been excluded by control experiments in which no effects were found following bilateral dorsal rhizotomies of the hindlimb enlargement of the spinal cord.
The present study suggests that, during two sensitive periods within the turtle swim cycle, muscle afferent input has direct access to the central nervous system (CNS) circuitry responsible for controlling locomotion. This conclusion is based on the following reported results. Muscle-nerve stimulation during the sensitive periods altered the period of the cycle in which the stimulus was delivered, and thus advanced or delayed the onset of the subsequent swim cycle. While the period of the perturbed cycle was changed, normal timing relationships were observed between muscles participating in the locomotor output. That is, muscle-nerve stimulation produced in-phase changes in duration or interburst interval which served to maintain the phase relationships between muscles, and therefore to preserve the intracycle motor pattern. These findings are consistent with input resulting from muscle-nerve stimulation during the sensitive periods having access to either the neuronal networks participating in patterning the locomotor output or to a CNS level prior to the pattern generating circuitry. Stimulation of the muscle-nerve during the sensitive periods was also found to permanently phase-shift the locomotor rhythm. The alteration in the onset times of all swim cycles subsequent to stimulus delivery represents a long-term change produced by a single 0.1 - to $0.3-\mathrm{msec}$ stimulus pulse. The permanent phase shift of the locomotor rhythm is consistent with input resulting from muscle-nerve stimulation during the sensitive periods having direct access to the neuronal networks responsible for controlling the cycle-to-cycle timing of the locomotor rhythm.

While the present study has identified periods within the swim cycle during which central timing networks are sensitive to electrical stimulation of muscle afferents, the role of naturally occurring proprioceptive information during the sensitive periods is not known. Previous reports have proposed that proprioceptive input in the form of positional (Grillner and Rossignol, 1978) and/or load (Pearson and Duysens, 1976) information may play an important role in signaling the appropriate time for transitions between retraction and protraction movements. In the turtlc, the transition from retraction to protraction is preceded by the SPA and the protraction/retraction transition follows the SPD. If peripheral feedback indicating that the limb has reached a transition point represents afferent information which is utilized by central timing networks, then muscle-nerve stimulation during the sensitive periods may be providing "misleading" timing information.

Limb position based on muscle length represents one type of proprioceptive information that could be communicated to central timing networks. For instance, the transition from retraction to protraction may be signaled by passive stretching of ambiens with its concomitant increase in muscle spindle discharge. An additional input, such as might be imposed by stimulation during the SPA, could shorten the swim cycle by incorrectly signaling the completion of the returnstroke. This signal could take the form of increased drive to the central timing network. The transition from protraction to retraction may be signaled by a decreased discharge rate in ambiens muscle spindles. Ambiens is contracting and presumably shortening at the end of the returnstroke. Muscle-nerve stimulation during the SPD may prolong or temporarily halt the swim cycle by signaling a failure to reach the desired completion of the returnstroke. This signal could take the form of an atypical increase in the drive to the central timing networks.

Load detection and compensation provide another, but not mutually exclusive, explanation of the possible relation of SPD stimulation to changes in the period of the swim cycle. The skeletofusimotor organization of motor neurons in the turtle is thought to be wellsuited for load compensation. Muscle-nerve stimulation during the SPD may mimic the increased muscle spindle input which would arise from an active contraction and intrafusal drive at a time when an obstacle or unanticipated resistance from water currents obstructs knee extension and the completion of the returnstroke. Although it is possible to interpret the findings of the present study in terms of peripheral feedback of load and positional cues, elucidation of the specific contributions of proprioceptive information 
during the sensitive periods will require future elaboration of the timing and type of information encoded by turtle limb afferents during swimming. It will also be important to assess the effects of stimuli delivered to the muscle-nerves innervating muscles active at different times within the locomotor cycle, such as the hip retractor/ knee flexors which participate in the turtle powerstroke.

Cutaneous stimulation during the sensitive periods produced changes in swim cycle period without perturbing the normal timing relationships between muscles participating in the locomotor output. Therefore, cutaneous as well as muscle afferents seem to have access during the sensitive periods to either pattern generating networks or to a prepattern generating level of the CNS. However, in contrast to the permanent phase shifts which resulted from muscle-nerve stimulation, cutaneous stimuli delivered during the sensitive periods caused a temporary phase shift of the swimming rhythm. Cutaneous stimuli during the SPA shortened the perturbed cycle and led to a phase advance which gradually disappeared due to a lengthening of poststimulus cycles. Perturbed cycle period increases, due to cutaneous stimulation during the SPD, were followed by a shortening of poststimulus cycles. Duysens (1977) found that cutaneous stimulation could cause rhythm resetting of cat stepping. In the Duysens study, the rhythm reset may have been permanent (although data are only reported for an ankle extensor and flexor and for two poststimulus step cycles), and there were no clear compensatory changes in cycle subsequent to the stimulation. Differences between the two studies are probably related to experimental design. Duysens used a premammilary cat with three limbs spontaneously locomoting on a treadmill, and trains of stimuli delivered to a cutaneous nerve of the fourth limb which was fixed to a frame. The temporary phase shifts found in the turtle were obtained by employing single stimulus pulses delivered to a preparation in which there were neither limb fixation nor movement-related influences from other limbs. The eventual return to the expected swimming rhythm following cutaneous nerve stimulation suggest that cutaneous inputs can modulate the locomotor rhythm, but that they do not have access to the central timing circuitry for locomotion.

Functional models of the neuronal networks controlling locomotion have typically proposed a single circuit whose oscillations are responsible for both patterning the motor output for each locomotor cycle and generating the cycle-to-cycle timing of the locomotor rhythm (see Grillner, 1977). The differential effects of muscle-nerve and cutaneous stimuli on the locomotor rhythm found in the present study have prompted the formulation of a new model for the functional organization of the neuronal networks subserving turtle swimming (Fig. 9). In order to explain the temporary locomotor phase shifts resulting from cutaneous nerve stimulation, the locomotor circuit has been subdivided into an oscillating central timing network (CTN) and a functionally separate intracycle central pattern generating network (CPG). In the proposed model, the CTN would be responsible for initiating each swim cycle, and therefore would organize the locomotor rhythm. The CPG would produce the proper phase relationships between muscles within each swim cycle, and therefore would organize the intracycle motor pattern. Each oscillation of the CTN would drive the CPG to produce the locomotor output for one swim cycle. Furthermore, it is suggested that as the CTN frequency increases, the excitatory influence from the CTN to the CPG increases. Certain components of the locomotor output, such as powerstroke/returnstroke ratio and the percentage of the swim cycle in which a muscle is active, vary as a function of cycle period (Lennard and Stein, 1977). It is proposed that these relationships are incorporated into the circuitry of the CPG and are correlated with the level of excitatory drive from the CTN to the CPG. The CPG could itself be an oscillatory network which is entrained by the CTN, or a one-cycle pattern generator (or damped oscillator) which is triggered on a cycle-by-cycle basis by the CTN.

The separation of the proposed model into timing and pattern generating functions is based on two classes of results from the present study. (1) Stimulation of muscle and cutaneous afferents during the sensitive periods can alter swim cycle period without perturbing the intracycle motor pattern. This suggests that both types of inputs have access to the CPG or a prepattern generator CNS level. (2) Stimulation of muscle afferents during the sensitive periods caused permanent phase shifts of the locomotor rhythm, while cutaneous inputs produced only temporary phase shifts. During a permanent phase shift, only the period of the perturbed cycle is changed. This results in all subsequent swim cycles beginning at a time which is different from the time predicted for unperturbed swimming sequences. A permanent phase shift can be thought of as resetting a clock (i.e., the CTN) which resets the locomotor rhythm. The effects of muscle-nerve stimulation (Fig. 9, solid line) can be explained by increased drive (increased excitation and/or decreased inhibition) to the CTN during the SPA and decreased drive during the SPD. Input from muscle afferents would reset the CTN, alter the drive from the CTN to the CPG, and thus change only the period of the perturbed cycle. The temporary phase shift produced by cutaneous input involved both period changes in the perturbed cycle and compensatory period changes in several subsequent cycles. This resulted in an initial phase shift followed by a recovery of swim cycle times to values predicted for unperturbed swim sequences. The recovery suggests that the CTN was running at its normal rate and was not reset during cutaneous modulation of the locomotor rhythm. Instead, it is proposed that the comperisatory period changes following cutaneous inputs are due to a ramp-like enhancement (during the SPD) or inhibition (during the SPA) of the coupling between the CTN and the CPG. The magnitude of the effect would decline over the course of several swim cycles, eventually allowing the CTN to drive the CPG with a normal coupling phase, and thus at the predicted (i.e., unperturbed) time. The effects of cutaneous input (Fig. 9, dashed lines) can be explained by compensatory changes in CTN/CPG coupling which are preceeded by an initial change in the period of the perturbed swim cycle produced by direct cutaneous input to the CPG. Cutaneous input during the SPA would transiently increase the excitability level of the CPG during the perturbed swim cycle, while input during the SPD would decrease CPG excitability. The proposed model does not preclude the participation of proprioceptive and cutaneous information in reflex pathways at other levels including post-CPG neurons (e.g., Fig. 7) and motor neurons.

The data presented in this report stress the need to carefully examine the effects of sensory inputs during locomotion on both the intracycle motor pattern and the locomotor rhythm. This can best be accomplished in preparations where there is minimal masking of the effect by postural constraints and movement-related inputs from other limbs. The functional separation of central timing networks trom central pattern-generating networks represents a hypothesis which is readily amenable to future testing.

\section{References}

Akazawa, K. J. W. Aldridge, J. D. Steeves, and R. B. Stein (1982) Modulation of stretch reflexes during locomotion in the mesencephalic cat. J. Physiol. (Lond.) 329: 553-567.

Andersson, O., and S. Grillner (1981) Peripheral control of the cat's step cycle. I. Phase dependent effects of ramp-movements of the hip during "fictive locomotion." Acta Physiol. Scand. 113: 89-101.

Andersson, O., and S. Grillner (1983) Peripheral control of the cat's step cycle. II. Entrainment of the central pattern generators for locomotion by sinusoidal hip movements during "fictive locomotion." Acta Physiol. Scand. 118: $229-239$.

Andersson, O., H. Forssberg, S. Grillner, and M. Lindquist (1978) Phasic gain control of the transmission in cutaneous reflex pall iways lo motoneurones during "fictive" locomotion. Brain Res. 149: 503-507.

Crowe, A., and A. H. M. F. Ragab (1970a) The structure, distribution and innervation of spindles in the extensor digitorum brevis I muscle of the tortoise Testudo graeca. J. Anat. 106: 521-538.

Crowe, A., and A. H. M. F. Ragab (1970b) Studies on the fine structure of the capsular region of tortoise muscle spindles. J. Anat. 107: 257-269. During, M. von, and M. R. Miller (1979) Sensory nerve endings of the skin 
and deeper structures. In Biology of the Reptilia, C. Gans, R. G. Northcutt, and P. Ulinski, eds., Vol. 9, pp. 407-441, Academic Press, New York.

Duysens, J. (1977) Fluctuations in sensitivity to rhythm resetting effects during the cat's step cycle. Brain Res. 133: 190-195.

Duysens, J., and G. E. Loeb (1980) Modulation of ipsi- and contralateral reflex responses in unrestrained walking cats. J. Neurophysiol. 44: 10241037.

Duysens, J., and R. B. Stein (1978) Reflexes induced by nerve stimulation in walking cats with implanted cuff electrodes. Exp. Brain Res. 32: 213-224.

English, A. W., and P. R. Lennard (1982) Interlimb coordination during stepping in the cat: in-phase stepping and gait transitions. Brain Res. 245: 353-364.

Forssberg, $H$. (1979) Stumbling corrective reaction: A phase-dependent compensatory reaction during locomotion. J. Neurophysiol. 42: 936-953.

Forssberg, H., S. Grillner, and S. Russignol (1975) Phase-dependent reflex reversal during walking in chronic spinal cats. Brain Res. 85: 103-107.

Forssberg, H., S. Grilner, and S. Rossignol (1977) Phasic gain control of reflexes from the dorsum of the paw during spinal locomotion. Brain Res. 132: $121-139$.

Grillner, S. (1975) Locomotion in vertebrates; central mechanisms and reflex interaction. Physiol. Rev. 55: 247-304

Grillner, S. (1977) On the neural control of movement-a comparison of different basic rhythmic behaviors. In Function and Formation of Neuronal Systems, G. S. Stent, ed., pp. 197-224, Dahlem Konferenzen, Berlin.

Grillner, S., and S. Rossignol (1978) On the initiation of the swing phase of locomotion in chronic spinal cats. Brain Res. 146: 269-277.

Guthe, K. F. (1981) Reptilian muscle: fine structure and physiological parameters. In Biology of the Reptilia, C. Gans and T. S. Parsons, eds., Vol. 11 pp. 265-354, Academic Press, New York.

Lennard, P. R. (1983) Afferent perturbations during controlled locomotion in the turtle. Soc. Neurosci. Abstr. 9: 635.

Lennard, P. R., and P. S. G. Stein (1977) Swimming movements elicited by electrical stimulation of the turtle spinal cord. I. The low spinal and the intact preparations. J. Neurophysiol. 40: 768-778

Matsukawa, K., H. Kamei, K. Minoda, and M. Udo (1982) Interlimb coordination in cat locomotion investigated with perturbation. I. Behavioral and electromyographic study on symmetric limbs of decerebrate and awake walking cats. Exp. Brain Res. 46: 425-437.

Naeije, M., and A. Crowe (1974) The response of chelonian muscle spindles to mechanical stimulation. Life Sci. 15: 131-136.

Naeije, M., and A. Crowe (1977) Responses of chelonian muscle spindles to mechanical stretch and fusimotor stimulation. J. Nel urophysiol. 40:814821.

Pearson, K. G., and J. Duysens (1976) Function of segmental reflexes in the control of stepping in cockroaches and cats. In Neural Control of Locomotion, R. M. Herman, S. Grillner, P. S. G. Stein, and D. G. Stuart, eds., pp. 519-537, Plenum Press, New York.

Rossignol, S., C. Julien, and L. Gauthier (1981) Stimulus-response relationships during locomotion. Can. J. Physiol. Pharmacol. 59: 667-674.

Schomburg, E. D., and H. B. Behrends (1978) The possibility of phasedependent monosynaptic and polysynaptic la excitation to homonymous motoneurons during fictive locomotion. Brain Res. 143: 533-537.

Shik, M. L., F. V. Severin, and G. N. Orlovsky (1966) Control of walking and running by means of electrical stimulation of the mid-brain. Biophysics 11: 756-765.

Stein, P. S. G. (1978) Motor systems, with specific reference to the contro of locomotion. Annu. Rev. Neurosci. 1:61-81.

Stein, P. S. G., G. A. Robertson, J. Keifer, M. L. Grossman, J. A. Berenbeim, and P. R. Lennard (1982) Motor neuron synaptic potentials during fictive scratch reflex in turtle. J. Comp. Physiol. 146: 401-409.

Walker, W. F., Jr. (1973) The locomotor apparatus of Testudines. In Biology of the Reptilia, C. Gans and T. S. Parson, eds., Vol. 4, pp. 1-100, Academic Press, New York.

Wand, P., A. Prochazka, and K. H. Sontag (1980) Neuromuscular responses to gait perturbations in freely moving cats. Exp. Brain Res. 38: 109-114.

Wetzel, M. C., and D. G. Stuart (1976) Ensemble characteristics of cat locomotion and its neural control. Prog. Neurobiol. 7: 1-98. 